\title{
THREE-DIMENSIONAL HIERARCHICAL MICROBATTERY ELECTRODES FOR TUNABLE HIGH ENERGY AND POWER DENSITY BATTERIES
}

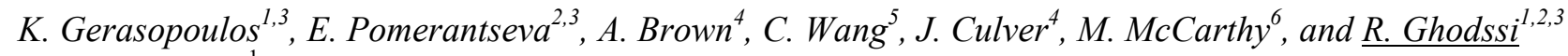 \\ ${ }^{1}$ MEMS Sensors and Actuators Laboratory, Institute for Systems Research; \\ ${ }^{2}$ Department of Electrical and Computer Engineering; \\ ${ }^{3}$ Department of Materials Science and Engineering; \\ ${ }^{4}$ Department of Plant Sciences and Landscape Architecture; \\ ${ }^{5}$ Department of Chemical and Biomolecular Engineering \\ University of Maryland, College Park, MD 20742, USA \\ ${ }^{6}$ Department of Mechanical Engineering and Mechanics, Drexel University, Philadelphia, PA 19104, USA
}

\begin{abstract}
We present a novel approach for the fabrication of hierarchical electrodes that integrates bottom-up nanostructure self-assembly and top-down microfabrication processes. The electrodes consist of virus-templated nanostructures self-assembled on three-dimensional micropillars. Active battery material $\left(\mathrm{V}_{2} \mathrm{O}_{5}\right)$ is conformally deposited using atomic layer deposition (ALD) on the hierarchical micro/nano network. Electrochemical testing of these electrodes indicates a 3 -fold increase in energy density compared to nanostructures alone, while maintaining the high power characteristics. Investigation of capacity scaling for varying active material thickness reveals underlying limitations and highlights the importance of our method in controlling both energy and power density with structural hierarchy.
\end{abstract}

\section{INTRODUCTION}

Three-dimensional micro/nano electrodes have been extensively researched as components for next-generation microbatteries [1]. Microstructures can increase material loading for the same device footprint, but lack in power density due to micrometer-size diffusion lengths. Nanostructures enable faster reaction kinetics but the electrode porosity reduces active material mass, thus limiting the available energy density. While the advantages of micro/nano structures have been independently demonstrated, no architecture integrating both components has been presented. In this work, the benefits of both length scales are combined for the first time, through the integration of virusstructured nanomaterials and 3-D micropillars. The experimental results show tunable energy density scaling (due to microstructures) and retention of high rate performance/power density (due to the nanostructures).

The key enabling technology in our approach is the use of the Tobacco mosaic virus (TMV) as a template for the synthesis of nanostructured materials. The TMV is a cylindrical plant virus which can be genetically modified to facilitate electroless metal coating and self-assembly onto various surfaces. The metallic thin film encasing the virus is uniform, highly conductive, and enables the rigidity of the resulting 3D nano-network. Previously we have demonstrated core/shell virus-templated Li-ion battery electrodes as well as photolithographic patterning and 3-D assembly of the particles [2-5]. These capabilities enable fabrication on substrates compatible with standard semiconductor processes, which is important factor in our approach. In this work, hierarchical electrodes are fabricated and their energy and power density are for the first time characterized.

\section{FABRICATION}

The electrodes were fabricated on silicon wafers with an evaporated gold seed layer. A three-spin process in AZ9260 photoresist was employed to form a $65 \mu \mathrm{m}$ thick polymer mold.
Gold pillars with $20 \mu \mathrm{m}$ diameter were electroplated from a commercial sulfite-based solution. Based on the dimensions in this design, a three-fold increase in area can be achieved. After electrodeposition, the photoresist was removed in acetone and the micropillars were coated with the metalized TMV template as described previously [2-5]. Finally, $\mathrm{V}_{2} \mathrm{O}_{5}$ layer was deposited using ALD at $170^{\circ} \mathrm{C}$. The electrodes fabrication process is schematically illustrated in Figure 1. Electrodes with and without micropillars were tested to investigate the capacity increase in the hierarchical design at various current densities and rates as well as different thicknesses of active materials.

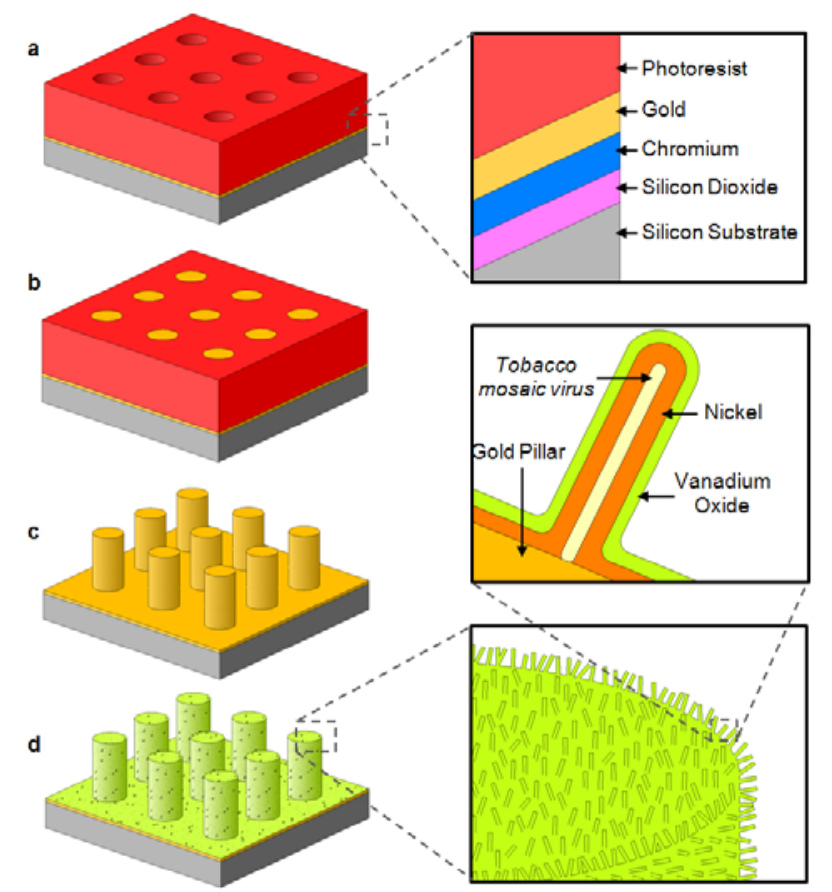

Figure 1: Schematic representation of the hierarchical electrode fabrication: (a) the photoresist mold is patterned on the silicon wafer (the inset shows the full layer structures); (b) gold is electroplated through the mold; (c) the mold is dissolved in an acetone bath; (d) TMV self-assembles onto the chip surface and then coated with $\mathrm{Ni}$ and $\mathrm{V}_{2} \mathrm{O}_{5}$; insets show the textured micro/nano structure of the electrode and an individual viral nanorod.

\section{RESULTS}

Figure 2 shows SEM and TEM images of the hierarchical electrodes with very uniform TMV coating of gold micropillars. The highly porous 3D nanonetwork is formed by virus particles (Fig. 2c). Figure $2 \mathrm{~d}$ shows a cross-section TEM image taken from the sidewall of the pillar demonstrating that the $\mathrm{V}_{2} \mathrm{O}_{5}$ coating is 
uniform regardless of the orientation of the particle. This is a significant attribute of ALD, which is ideally suited to create uniform, conformal coatings of complex 3D geometries with precise control over film thickness.

Figure 3a shows discharge/charge curves for electrodes with and without micropillars. The thickness of $\mathrm{V}_{2} \mathrm{O}_{5}$ in these experiments was $30 \mathrm{~nm}$ (1000 ALD cycles). The capacity for the hierarchical electrode is $38 \mu \mathrm{Ah} \mathrm{cm} \mathrm{cm}^{-2}$, while the nanostructured only electrode exhibits a capacity of $13 \mu \mathrm{Ah} \mathrm{cm}^{-2}$. This is in excellent agreement with the increase in mass and surface area. As indicated in Figure 3b, where the capacity versus cycle number for the first 35 cycles of operation is plotted. This 3-fold increase in energy density is demonstrated throughout the course of testing without batteries failure.

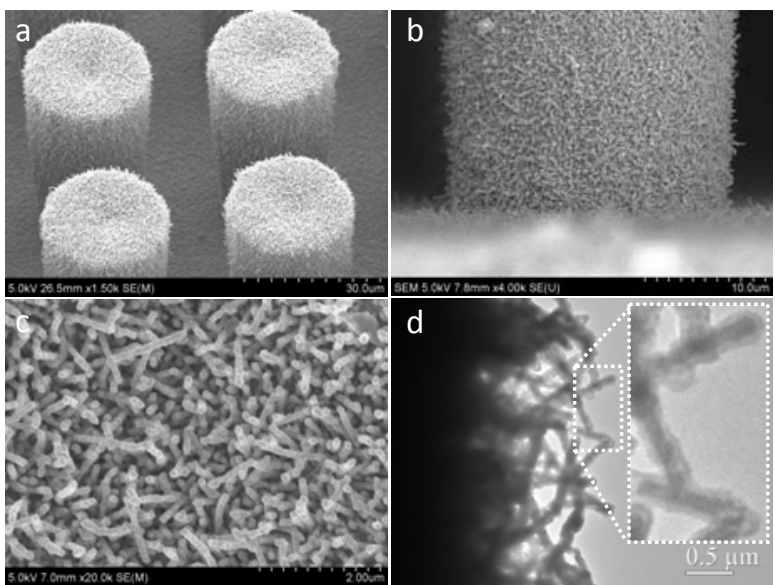

Figure 2: SEM and TEM images of the hierarchical electrodes: (ab) micropillars coated with the $\mathrm{TMV} / \mathrm{Ni} / \mathrm{V}_{2} \mathrm{O}_{5}$ layers $\left(\mathrm{V}_{2} \mathrm{O}_{5}\right.$ was deposited for 1000 ALD cycles); (c) close-up image showing the top of micropillar; (d) the sidewall of a micropillar, showing uniform $\mathrm{V}_{2} \mathrm{O}_{5}$ coverage regardless TMV particles orientation.
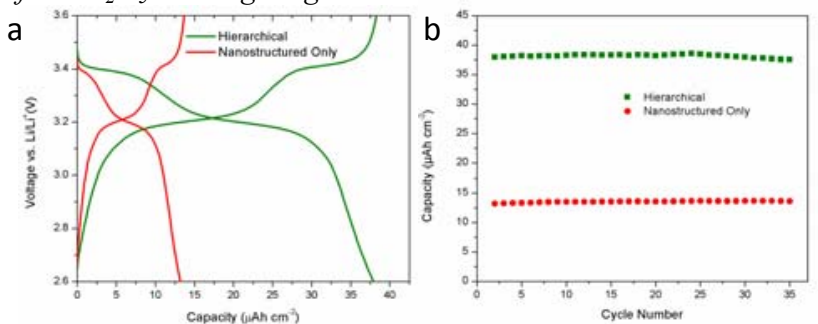

Figure 3: Electrochemical characterization of virus-structured electrodes with and without micropillars (a) galvanostatic charge/discharge curves at a current of $10 \mu \mathrm{A}$ (electrode footprint area was $1.1 \mathrm{~cm}^{2}$ ); (b) capacity vs. cycle number for the samples used in a for the first 35 cycles of operation.
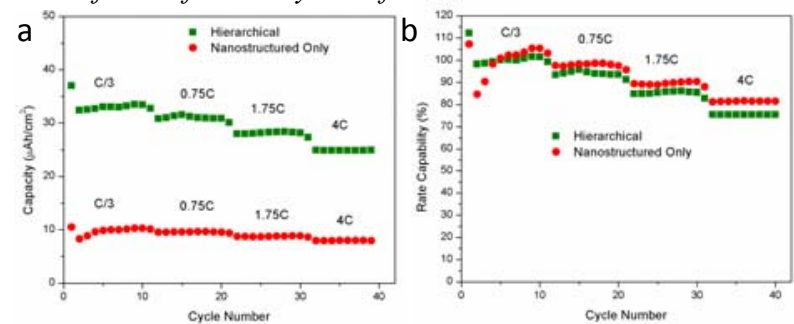

Figure 4: Rate capability data at different C-rates for hierarchical and nanostructured only electrodes: (a) capacity vs. cycle number; (b) capacity retention vs. cycle number (the data is presented as the ratio of $C_{i} / C_{\text {inav }}$, where $C_{i}$ is the capacity for each cycle, and $C_{\text {inav }}$ is the average initial capacity for the lower $C$-rate).
The rate performance results are shown in Figure 4. In these tests, electrodes were cycled in increments of 10 cycles for progressively increasing current values. The capacity remains 3 times higher for the hierarchical electrodes for all C-rates (Fig. 4a), while both types of electrodes demonstrate similar rate capabilities (Fig. 4b). This result indicates that the addition of micropillars increases the energy density of the electrodes without affecting the high rate performance. The morphology and discharge/charge curves for hierarchical electrodes with different $\mathrm{V}_{2} \mathrm{O}_{5}$ thicknesses are shown in Figure 5. Interestingly, the capacity increase exhibits a non-linearity as the thickness of the active material increases from $\sim 60 \mathrm{~nm}$ to $\sim 120 \mathrm{~nm}$ (Fig. $5 \mathrm{~d}$ ). This behavior is caused by progressive blocking of open spaces between closely-packed adjacent TMV nanorods with increasing deposition thickness (Fig. 5e). This identifies inherent limitations in increasing nanostructure thickness and highlights the importance of our technology for independently controlling energy and power density.
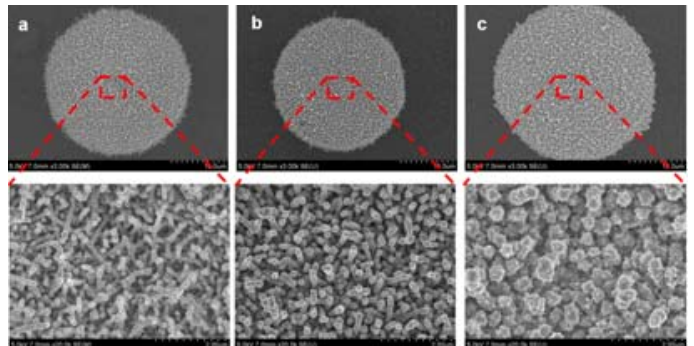

d
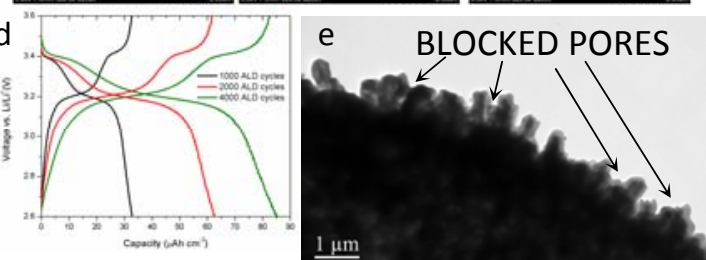

Figure 5: Hierarchical electrode morphology and electrochemical performance for different $\mathrm{V}_{2} \mathrm{O}_{5}$ thickness: (a-c) SEM images showing the top of micropillars for 1000, 2000 and 4000 ALD cycles of $\mathrm{V}_{2} \mathrm{O}_{5}$, respectively; (d) galvanostatic discharge/charge curves for hierarchical electrodes at a current of $12 \mu \mathrm{A} ; V_{2} \mathrm{O}_{5}$ was deposited for 1000, 2000, and 4000 ALD cycles, aiming at thicknesses of $30 \mathrm{~nm}, 60 \mathrm{~nm}$, and $120 \mathrm{~nm}$, respectively; (e) cross section TEM image taken from the sidewall of micropillar for the sample with $4000 \mathrm{ALD}$ cycles of $\mathrm{V}_{2} \mathrm{O}_{5}$.

\section{REFERENCES}

[1] T.S. Arthur, D.J. Bates, N. Cirigliano, D.C. Johnson, P. Malati, J.M. Mosby, E. Perre, M.T. Rawls, A.L. Prieto, B. Dunn, "Three-dimensional electrodes and battery architectures", MRS Bulletin, 36, 523 (2011).

[2] K. Gerasopoulos, M. McCarthy, P. Banerjee, X. Fan, J.N. Culver, and R. Ghodssi, "Biofabrication methods for the patterned assembly and synthesis of viral nanotemplates", Nanotechnology 21, 055304 (2010).

[3] K. Gerasopoulos, X. Chen, J. Culver, C. Wang, and R. Ghodssi, "Self-assembled $\mathrm{Ni} / \mathrm{TiO}_{2}$ nanocomposite anodes synthesized via electroless plating and atomic layer deposition on biological scaffolds", Chem. Comm. 46, 7349 (2010).

[4] E. Pomerantseva, K. Gerasopoulos, X. Chen, G. Rubloff, and R. Ghodssi, "Electrochemical performance of the nanostructured biotemplated $\mathrm{V}_{2} \mathrm{O}_{5}$ cathode for lithium-ion batteries", J. Power Sources 206, 282 (2012).

\section{CONTACT}

Professor Reza Ghodssi: Tel.: +1 301405 8158; E-mail: ghodssi@umd.edu 